\title{
Succession in the Caecal Microbiota of Developing Broilers Colonised by Extended-spectrum $\beta$ - lactamase-producing Escherichia Coli
}

Ingrid Cardenas-Rey

Wageningen Bioveterinary Research

Teresita Bello Gonzalez

Wageningen Bioveterinary Research

Jeanet Van der Goot

Wageningen Bioveterinary Research

Daniela Ceccarelli

Wageningen Bioveterinary Research

Gerwin Bouwhuis

Gezondheidscentrum voor pluimvee

Danielle Schillemans

Wageningen Bioveterinary Research

Stephanie Jurburg

German Centre for Integrative Biodiversity Research (iDiv) Halle-Jena-Leipzig

Kees Veldman

Wageningen Bioveterinary Research

Arjan De Visser

Wageningen UR PRI: Wageningen University and Research Wageningen Plant Research

Michael Brouwer ( $\square$ mike.brouwer@wur.nl)

Wageningen Bioveterinary Research https://orcid.org/0000-0002-1067-7555

\section{Research Article}

Keywords: Broilers, ESBL, Escherichia coli, microbiota

Posted Date: September 27th, 2021

DOI: https://doi.org/10.21203/rs.3.rs-929974/v1

License: (c) (1) This work is licensed under a Creative Commons Attribution 4.0 International License.

Read Full License 
Succession in the caecal microbiota of developing broilers colonised by Extended-spectrum $\beta$ lactamase-producing Escherichia coli

Ingrid Cardenas Rey ${ }^{1,5}$, Teresita d.J. Bello Gonzalez ${ }^{1}$, Jeanet van der Goot ${ }^{1}$, Daniela Ceccarelli ${ }^{1}$, Gerwin Bouwhuis $^{2}$, Danielle Schillemans ${ }^{1}$, Stephanie Jurburg ${ }^{3,4}$, Kees T. Veldman ${ }^{1}$, J. Arjan G.M. de Visser ${ }^{5}$, Michael S. M. Brouwer ${ }^{1 \#}$

Affiliations:

${ }^{1}$ Dept. of Bacteriology, Host Pathogen Interactions and Diagnostics Development, Wageningen Bioveterinary Research, Lelystad, The Netherlands.

${ }^{2}$ Gezondheidscentrum voor Pluimvee, Emmen, The Netherlands.

${ }^{3}$ German Centre for Integrative Biodiversity Research (iDiv) Halle-Jena-Leipzig, Deutscher Platz 5e, 04103 Leipzig, Germany.

${ }^{4}$ Institute of Biology, Leipzig University, Deutscher Platz 5e, 04103 Leipzig, Germany.

${ }^{5}$ Laboratory of Genetics, Wageningen University and Research, Wageningen, The Netherlands.

\#Corresponding author:

Michael Brouwer

Department of Bacteriology and Epidemiology

Wageningen Bioveterinary Research

Wageningen University and Research

Tel: $+31-320238327$

Email:mike.brouwer@wur.nl 
Abstract

Background: Broilers are among the most common and dense poultry production systems, where antimicrobials have been used extensively to promote animal health and performance. The continuous usage of antimicrobials has selected for resistant bacteria, such as e Extended-spectrum $\beta$-lactamaseproducing Escherichia coli (ESBL-Ec). Here, we studied the ESBL-Ec prevalence and successional dynamics of the caecal microbiome of developing broilers in a commercial flock during their production life. Broilers were discriminated as ESBL-Ec colonised or not by selective culturing. Using 16S rRNA gene sequencing, we compared the richness, evenness and composition of the cecal microbiota of both broiler groups and assessed the combined role of age and ESBL status on the microbiota.

Results: We observed a linear trend in the proportions of ESBL-Ec throughout the broilers' production round, $X^{2}(1, N=12)=28.4, p<.001$. Over time, microbial richness was consistently higher in ESBL-Ec free broilers, but significant differences between both groups were found exclusively on day three (Wilcoxon rank-sum test, $p=.016)$. Bray-Curtis distance-based RDA (BC- dbRDA) showed no explanatory power of ESBL status, while age explained $14 \%$ of the compositional variation of the caecal microbiome, $F(2,66)=6.47, p=.001$.

Conclusions: This study assessed the role of ESBL-producing E.coli in the successional dynamics of the cecal microbiome in developing broilers and shows that the presence of ESBL-producing E.coli is

Keywords: Broilers, ESBL, Escherichia coli, microbiota. 
Poultry is one of the most important animal protein sources and are a major driver of the global growth in protein production (http://www.fao.org/3/a-i7465e.pdf). At the same time, the poultry industry has been identified as an important source of antimicrobial resistance (AMR; Saliu et al., 2017). Broiler chickens are among the most common and dense poultry production systems, where antimicrobials have been used extensively to promote animal health and performance. However, growth promotors have been banned since 2006 in the Netherlands and other countires since then. Nonetheless, the continuous therapeutic usage of antimicrobials has selected for AMR, such as extended-spectrum beta-lactamases (ESBL), among pathogenic and commensal bacteria [2].

ESBLs are recognised as a public health threat due to their capacity to inactivate extended-spectrum cephalosporins (ESC), a large group of critically important antibiotics belonging to the group of $\beta$-lactams $[3,4]$. ESBLs have emerged globally, particularly within members of the order of Enterobacterales, especially Escherichia coli $[5,6]$. The spread of ESBLs has been associated with conjugative plasmids capable of persisting in the host and the environment efficiently [7]. Favourable, nutrient-rich environments like the chicken's caeca enable commensal and pathogenic bacteria to exchange ESBL plasmids [8].

The development of the caecal microbiota of commercial broiler chickens has been associated primarily with environmental sources $[9,10]$. Immediately after hatching, chickens are colonised by exogenous microorganisms, including E. coli [11-13]. E. coli are common ESBL carriers that can potentially spread and act as a reservoir of these resistance genes in the caecal microbiota of broilers (Dame-Korevaar et al., 2019; Jiménez-Belenguer et al., 2016). As the broiler ages, the caecal microbiota undergoes successional changes, which involve rapid and directional turnover towards a more diverse community with a lower abundance of Enterobacterales, including E. coli [13]. How the timing of colonisation of ESBL-producing E. coli (ESBL-Ec) is affected by these successional dynamics, and whether the presence of ESBL-Ec affects the development of the cecal microbiota of broilers is unknown.

Here, we studied the ESBL-Ec prevalence and successional dynamics of the caecal microbiome of developing broilers in a commercial flock during their production life. Broilers were discriminated as ESBL-Ec colonised or not by selective culturing. Using 16S rRNA gene sequencing, we compared the richness, evenness and composition of the cecal microbiota of both broiler groups and assessed the combined role of age and ESBL status on the microbiota. We observed a linear trend in the proportions of ESBL-Ec throughout the broilers' production round. Over time, microbial richness was consistently higher 
in ESBL-Ec free broilers, but significant differences between groups were found exclusively on day three. Bray-Curtis distance-based RDA (BC- dbRDA) showed no explanatory power of ESBL status, while age explained $14 \%$ of the compositional variation of the caecal microbiome.

\section{Results}

\section{Prevalence and typing of ESBL producing $E$. coli}

Caecal samples were collected on a Dutch commercial broiler farm throughout the production round (days $0-7,14,21,28$ and 35). Culture-based methods were applied to each caecal sample to discriminate between ESBL-Ec colonised $\left(E S B L-E c^{+}\right)$and non-colonised (ESBL-Ec-) broilers. ESBL-Ec were detected from day 2 with a sample prevalence of $11 \%$ CI $[1 ; 34]$ and rapidly increased to $72 \%$ CI [46; 90] until day 5. Prevalence fluctuated between days 6 and 28 but remained above 50\%. All caecal samples were positive for ESBL-Ec on day 35 (Fig. 1a). A linear trend in the proportions of ESBL-Ec was observed throughout the broilers' production round, $X^{2}(1, N=12)=28.4, p<.001$.

Real-time PCR was used to determine the most prevalent ESBL/AmpC-gene families responsible for the ESC-resistant phenotype in the Netherlands, CTX-M, SHV, TEM and CMY. Both blashv and blatem PCR products were amplified for all 109 ESC-resistant E. coli isolates. Sanger sequencing of 18 of these products spread over the production round indicated that all strains carry the ESBL gene blasHV-12 and the non-ESBL beta-lactamase blaTEM-1b (see Table S1). Transformation and subsequent PCR-based replicon typing (PBRT) demonstrated that blasHV-12 genes were located on IncI1a plasmids in all 18 isolates. From these, ten isolates spread over the production round were chosen for in-depth analysis. PCR and Sanger sequencing showed that all ten isolates belonged to the multilocus sequence typing (MLST) type 1011, while all IncI1a plasmids belonged to PMLST type 3.

\section{Characterisation of the caecal bacterial composition of ESBL-Ec ${ }^{+}$and ESBL-Ec broilers $^{-}$}

After quality control, 2,582,888 16S rRNA gene sequences were recovered from eighty-nine caecal samples and rarefied to 29,351 reads per sample. One sample (day zero) with a low number of reads $(<30)$ was excluded from the analyses. Due to the rapid increase of ESBL-Ec prevalence over time, microbiota comparisons between ESBL-Ec ${ }^{+}$and ESBL-Ec- broilers were only possible between days 3 and 28. Except for two phyla, no significant differences in the relative abundances of phyla were found between broilers' groups. Bacteroidetes was significantly higher in ESBL-Ec ${ }^{+}$broilers on day 21 (ANCOM- 
$\mathrm{BC}, p=.003$ ), while Tenericutes were significantly higher in ESBL-Ec- broilers on day 28 (ANCOM-BC, $p$ $<.001)$. Both phyla represented only a small part of the total community $(<5 \%)$.

Of the six bacterial phyla identified, Firmicutes were the most abundant with an average relative abundance of $77.9 \% \pm 20.3$, followed by Proteobacteria $(18.2 \% \pm 22.0)$ and Bacteroidetes $(3.1 \% \pm$ 6.3). Across all samples, Actinobacteria, Tenericutes and Cyanobacteria combined represented only $0.8 \%$ \pm 0.2 of the total taxa. Firmicutes and Proteobacteria predominated in the developing caecal microbiota of ESBL-Ec ${ }^{+}$and ESBL-Ec- broilers until day 14 (Fig. 2a). While the relative abundances of these phyla fluctuated during this period, no significant differences over time were found (ANCOM-BC, $p>.05$ ). Bacteroidetes were detected from day 14 onwards with a continuous increase in numbers until the end of the broiler's production life $(16.6 \% \pm 5.1)$.

Shifts in relative abundances of families were observed over time (Supplementary Fig. 1). However, no differential abundance was found between ESBL-Ec ${ }^{+}$and ESBL-Ec broilers (ANCOM-BC, $p>.05$ ). Within the phylum Firmicutes, families Ruminococcaceae and Lachnospiraceae were the most prevalent over time in both broiler groups. Proteobacteria and Bacteroidetes were represented by the families Enterobacteriaceae (99\%) and Rikenellaceae (100\%), respectively. Actinobacteria's main representative families were Eggerthellaceae and Bifidobacteriaceae, while for Tenericutes, it was Anaeroplasmataceae.

The ten most abundant genera in both broiler groups made up $45.7 \% \pm 1.4$ and $52.9 \% \pm 2.7$ of the total microbial community of ESBL-Ec ${ }^{+}$and ESBL-Ec- broilers, respectively. Only on day three, the bacterial community of ESBL-Ec ${ }^{+}$broilers was dominated by five genera; Klebsiella, Lactobacillus, Enterococcus, Blautia and Escherichia/Shigella (87\% of total the whole community), while in ESBL-ECbroilers, the bacterial composition was much more diverse (Fig. 2c). The relative abundances of Eisenbergiella and Subdoligranulum were significantly higher in ESBL-Ec- broilers on days 3 and 4, respectively $(\mathrm{ANCOM}-\mathrm{BC}, p<.001)$. Alistipes was the most abundant taxon in ESBL-Ec ${ }^{+}$broilers on days $21(13.1 \% \pm 5.5)$ and $28(14.5 \% \pm 5.4)$. Significant differences were found only on day $21 ;$ ESBL-EC $^{-}$ broilers made up only $3.8 \% \pm 2.2$ of the community (ANCOM-BC, $p=0.01$ ).

\section{Relative abundance of Escherichia/Shigella}

Fifteen amplicon sequence variants (ASVs) associated with Escherichia/Shigella were recovered (Supplementary Fig. 2), but only two persisted over time. Across all samples, Escherichia/Shigella was dominant both in ESBL-Ec ${ }^{+}(6.5 \% \pm 7.1)$ and ESBL-Ec- broilers $(17.7 \% \pm 19.3)$, but they decreased over time, from $37.3 \% \pm 24.0$ on day 1 , to $1.3 \% \pm 2.2$ on day 35 . Importantly, on day 1 , all chickens were ESBL free. The relative abundance of Escherichia/Shigella decreased as broilers aged. No 
differential abundance of Escherichia/Shigella between ESBL-Ec ${ }^{+}$and ESBL-Ec- broilers were observed over time (ANCOM-BC, $p>0.05$ )

\section{Alpha diversity}

\section{Microbial richness and evenness}

To study the richness and evenness of the cecal microbiota, we measured the number of observed ASV $\mathrm{S}$ and Pielou's evenness, respectively. Microbial richness increased with the broilers' age, from $103.3 \pm$ 115.2 ASVs on day zero to $649.6 \pm 202.9$ on day 35 (Fig. 3a). Broiler's age significantly predicted microbial richness over time with the formula; observed richness $=101.24+20.87 \times\left(\right.$ time), $\operatorname{Adj} R^{2}=$ $0.76, p<.001$. Over time, observed richness was consistently higher in ESBL-Ec- broilers (Fig. 3b), but significant differences between both groups were found exclusively on day three; ESBL-Ec- showed a higher number of ASVs than ESBL-Ec ${ }^{+}$broilers (Wilcoxon rank-sum test, $p=.016$ ).

Community evenness rapidly increased in young broilers (day 0-5) and plateaued after five days in both groups (Fig. 3b). No significant differences were found between ESBL-Ec ${ }^{+}$and ESBL-Ec- broilers (Wilcoxon rank-sum test, $p>.05$ ).

\section{Beta diversity}

Changes in the caecal microbiota were analysed with Bray-Curtis principal coordinate (BC-PCoA) and Bray-Curtis distance-based RDA (BC-dbRDA) analyses. Samples clustered significantly according to age (Fig. 4 ; Adonis, $p<.001$ ). Pairwise analysis showed significant differences between all days pairing (Pairwise Adonis, $p<.05$ ), except for days $0-1$ and days $28-35$. No significant clustering by ESBL-status was observed in samples from days 3-28. No collinearity was found between age and ESBL $(V I F<3)$. BC-dbRDA showed no explanatory power of ESBL status, while age explained $14 \%$ of the compositional variation of the caecal microbiome, $F(2,66)=6.47, p=.001$.

\section{Discussion}

Exploring the successional dynamics of the caecal microbiota of broilers can reveal windows of opportunity to implement intervention strategies that reduce the spread of resistant commensal and pathogenic bacteria. However, little is known about how changes in the developing caecal microbiota affect the prevalence of ESBL-Ec or how the presence of ESBL-Ec affects the microbiome. Here, we studied the caecal microbiota of developing commercial broilers in normal farming conditions and 
classified them as ESBL-Ec colonised and non-colonised. We found no clear divergence between these two groups over time, suggesting that the presence of ESBL-Ec does not have consistent effects on the cecal microbiome of developing broilers.

ESBL-Ec was detected on day two, and its prevalence increased rapidly over time. All broilers tested negative for ESBL-Ec on days zero and one, suggesting that the farm environment was the likely source for ESBL-Ec colonisation. The ESBL-gene, plasmid typing and MLST results were identical for all samples, suggesting a clonal spread of ESBL-Ec throughout the flock. The clonal distribution of ESBL-Ec has been associated with the high shedding of ESBL-Ec after colonisation in previous studies [14].

In contrast to the ESBL-Ec prevalence trends, the relative abundance of Escherichia/Shigella decreased over time in both groups of broilers. This reflects the broilers' microbiota dynamic development over time; as broilers age, the microbial diversity increases, causing shifts in bacterial abundances. Despite the continued dominance of members of the phylum Firmicutes, Escherichia/Shigella persisted throughout the broilers production round. Compositional changes over time resembled those documented in previous studies: the relative abundance of Proteobacteria decreased over time, while the relative abundance of Firmicutes and Bacteroidetes gradually increased (Jurburg et al., 2019; Ocejo et al., 2019; Richards., 2019).

A possible explanation for the high prevalence of ESBL-Ec in broilers at day 35 , when the relative abundance of Escherichia/Shigella was not higher than $5 \%$, is that other microbiota community members might acquire, carry and contribute to the spread of ESBL genes. A previous in vitro study reported the successful transfer of CMY-2-encoding IncI1 plasmids from exogenous $E$. coli to members of the human gut microbiota [18]. IncI1 plasmids have been described as promiscuous plasmids that can migrate within the members of the order Enterobacterales and likely between different bacterial species (Rozwandowicz et al., 2018; Wong et al., 2015). To better understand the prevalence of ESBL-Ec in the broilers' caecal microbiota, we intend to quantify $E$. coli (16S rRNA gene copy numbers) by quantitative PCR ( PPCR) in future studies.

Consistent with the literature (Jurburg et al., 2019; Ocejo et al., 2019), observed caecal microbial richness increased (linearly) as broilers aged in both groups. Overall, microbial richness was not significantly different between groups. However, a higher microbial richness was consistently observed in ESBL-Ec- compared to ESBL-Ec ${ }^{+}$broilers over time. In line with this finding, ESBL-colonised broilers showed a much less diverse microbial composition on day three, with only five genera representing $86 \%$ of the total community. These results suggest small but consistent alterations in the microbiome's 
competitive landscape, which could be further explored with controlled, culture-based laboratory experiments.

As shown in previous research (Jurburg et al., 2019; Kers et al., 2019; Ocejo et al., 2019; Takeshita et al., 2021), host ageing is one of the drivers of microbiota composition. In our study, age explained $14 \%$ of the microbial composition variability. The succession of the broilers' microbial communities was observed in three stages. The first stage (days 0-4) was dominated by Firmicutes and Proteobacteria, mainly by families Clostridiaceae, Enterococcaceae and Enterobacteriaceae. A decrease in Proteobacteria $(<10 \%)$ and a complete dominance $(>80 \%)$ by members of the Firmicutes (families Ruminococcaceae, Lachinospiraceae and Lactobacillaceae) characterised the second stage (days 5-14). In the third stage (days 21-35), Proteobacteria continued steadily decreasing (<5\%), while Bacteroidetes emerged, accounting for $13.7 \%$ of the total community. Firmicutes were still extensively represented principally by families Ruminococcaceae and Lachinospiraceae and the appearance of members of the Clostridiales. Despite the differences in study designs, these observations support the results from Jurburg et al. (2019) and Ocejo et al. (2019), in which successional dynamics were detected in 3 stages and represented by similar phyla and families.

On the other hand, the colonisation by ESBL-Ec did not explain any variability in the caecal composition in this study. These results match those observed by Ducarmon et al. (2021), in which asymptomatic gut carriage of ESBL-Ec was not associated with differences in the bacterial species composition in humans.

To the best of our knowledge, this is the first study assessing the differences in the microbiota composition and diversity of ESBL-Ec ${ }^{+}$and ESBL-Ec broilers from a commercial farm throughout the production round. A previous experimental study examined the microbiota of broilers colonised with ESBL-Ec and treated with competitive exclusion products on days 5 and 21. [24]. However, the study aimed to evaluate the effect of compartmentalisation and interventions on the transmission and prevention of ESBL-Ec colonisation in the broiler microbiota composition more than the effect of ESBL-Ec on the broilers microbiome. Longitudinal-experimental studies which manipulate ESBL-Ec prevalence could reveal the process of colonisation of ESBL-Ec in the cecal microbiome and shed light on the relationship between AMR bacteria, the caecal microbiome, and potential avenues for microbiome-based control of AMR. Our study highlights the need to consider the natural dynamics of the host-microbiome development and colonisation by resistant bacteria. 
This study contributes to assessing the role of ESBL-Ec in the successional dynamics of the cecal microbiome in developing broilers and shows that the presence of ESBL-Ec is associated with mild but consistent reductions in alpha diversity and transient compositional differences. Our study further documents the clonal spread of ESBLs and points at the farm environment as a likely source for ESBLs. Our study documents ESBL prevalence in a single location and during one growth cycle. Future research should aim to determine the universality of the patterns of ESBL-Ec spread that we have found across production cycles and farms and seek to more precisely understand whether the presence of ESBL modulates the competitive landscape of the broiler microbiome or vice-versa.

\section{Material and Methods}

\section{Ethics}

Ethical approval for this study's design was granted through the animal ethical commission at Wageningen University and Research from the Dutch central committee animal experiments in compliance with Dutch legislation, application number AVD4010020171486.

\section{Sample collection}

Broiler chickens (Gallus gallus domesticus) originated from a single flock in a Dutch commercial broiler farm. Fertilised eggs were hatched on the broiler farm. In total, 216 birds were randomly sampled at 12 different time points of the production cycle (days $0-7,14,21,28$, and 35 of age), beginning on the day of hatching. Eighteen chickens were euthanised for caecal sample collection at each time point. Samples were collected between October and December 2017. Caecal content was aseptically collected from individual birds. A portion was used for cultivation within 4 hours, while the rest of the sample was preserved for further analyses.

\section{Selective isolation of ESBL-E. coli}

Selective culture media were used for screening the caecal samples for the presence of ESBL-producing E. coli. Samples $(n=216)$ were aseptically collected from individual caeca by using sterile swabs. The collected swabs were placed on $3 \mathrm{ml}$ of peptone water for bacterial enrichment and incubated overnight at $37^{\circ} \mathrm{C}$. A day after, $10 \mu \mathrm{L}$ of the enrichment were inoculated on a MacConkey agar plate containing one $\mathrm{mg} / \mathrm{L}$ cefotaxime. After overnight incubation at $44^{\circ} \mathrm{C}$, a suspected $E$. coli colony was randomly selected, sub-cultured on Heart Infusion Sheep blood (5\%) agar (HIS) and incubated at $37^{\circ} \mathrm{C}$ overnight. Colonies were confirmed as E. coli by MALDI-TOF mass spectrometry (Maldi Biotyper Compass, Bruker () . 


\section{Plasmid identification}

Putative ESBL-E. coli isolates were selected for gene and plasmid identification using real-time PCR [25] and PCR Based Replicon Typing [26], respectively. Isolates were grown in Luria-Bertani (LB) broth and incubated overnight at $37^{\circ} \mathrm{C}$ (shaking). Plasmid DNA was extracted as previously described [27] and used for bacterial transformation. For this, plasmid DNA $(1 \mu \mathrm{L})$ was mixed with $12 \mu \mathrm{L}$ of electrocompetent cells (ElectroMax ${ }^{\mathrm{TM}} \mathrm{DH} 10 \mathrm{~B}$ cells, Gibco Invitrogen). The mix was added to a $0.1 \mathrm{~cm}$ gap length cuvette and kept on ice. Electroporation was performed using $200 \Omega-1.25 \mathrm{Kv}-25 \mu \mathrm{F}$. Cells were incubated at $37^{\circ} \mathrm{C}$ for 45 minutes on LB broth and then streaked on LB agar plates containing $1 \mathrm{mg} / \mathrm{L}$ cefotaxime. After overnight incubation $\left(37^{\circ} \mathrm{C}\right)$, one putative transformant colony was selected per plate and restreaked on selective LB agar. DNA of putative transformants was extracted for i. resistance gene confirmation and ii. plasmid identification by PCR Based Replicon Typing (PBRT 2.0 Diatheva) according to the manufacturer's recommendations.

\section{Nucleic Acid Extraction}

DNA was extracted from 0.2 grams of caecal content using the Qiagen kit QIAamp Fast DNA stool mini kit (Qiagen, Hilden, Germany). In addition to the manufacturer's protocol, a bead-beating step was included at the beginning of the extraction; samples were treated in a FastPrep- $245 \mathrm{G}^{\circledR}$ at $30 \mathrm{~Hz}$ for 30 seconds in each of the three cycles. The final elution of the DNA occurred in $35 \mu$ nuclease-free water. The ZymoBIOMICS Microbial Community Standard (Zymo Research) was used as positive control for the extraction and downstream processing steps.

\section{Amplification and 16S rRNA gene sequencing}

Extracted DNA was quantified using a CLARIOstar ${ }^{\circledR}$ (BMG Labtech, Ortenberg, Germany). The V3-V4 region of the $16 \mathrm{~S}$ rRNA gene was amplified in all the samples in triplicate to minimise PCR biases. The PCR reaction consisted of $25 \mu$ total volume, including $12.5 \mu$ of Q5 master mix, $1 \mu$ l of V3V4 primer mix (CVI_V3-forw 5' CCTACGGGAGGCAGCAG 3' and CVI_V4-rev 5' GGACTACHVGGGTWTCT 3'), 2.5 l DNA and $9 \mu \mathrm{l}$ of nuclease-free water. Amplification conditions consisted of $98^{\circ} \mathrm{C}$ for 2 min, 20 cycles at $98{ }^{\circ} \mathrm{C}$ for $10 \mathrm{sec}, 55^{\circ} \mathrm{C}$ for $30 \mathrm{sec}, 72^{\circ} \mathrm{C}$ for $10 \mathrm{sec}$, and finally $72{ }^{\circ} \mathrm{C}$ for $7 \mathrm{~min}$. PCR products were pooled, analysed on a 48 well $2 \%$ E-gel (ThermoFisher Scientific) and then sequenced on a Miseq (Illumina, San Diego, CA) using a 2×300 bp paired-end cycle sequencing run.

\section{Amplicon sequence variant (ASV) identification and taxonomy assignment}

Analyses of the 16S rRNA gene sequence reads were conducted in R 3.6.3 [28] with the dada2 v1.14.0 package [29]. Low-quality reads were filtered and trimmed using the parameters truncLenFR=240,240 
and trimLeft $=17$, respectively. After merging the forward and reverse reads and removing chimaeras, ASVs were assigned with the SILVA v.132 classifier [30].

\section{Downstream and statistical analysis}

Before downstream analysis, microbiome data were rarefied to 10875 reads per sample $(r n g s e e d=1)$. Alpha diversity (observed richness and evenness) was estimated at the ASV level in all samples using the phyloseq v.1.30.0 and microbiome v.2.1.26 packages, respectively. Differences in alpha diversity between broiler groups were evaluated by using Wilcoxon rank-sum test with the Benjamini-Hochberg $(\mathrm{BH})$ correction.

Broiler caecal microbiota relative abundance was estimated at phylum, family and genus level with the phyloseq v.1.30.0 package. Relative abundances are presented as mean and standard deviations. Differential abundance testing between broilers groups was performed for each time point using the ANCOMBC v.1.0.5 package with the Benjamini-Hochberg $(\mathrm{BH})$ correction [31].

Beta diversity (constrain and unconstrained) analyses were performed using the Bray-Curtis distances at the ASV level. Changes in the broiler's caecal microbial community composition were visualised with principal coordinate analysis (PCOA) and tested with permutational multivariate analysis of variance (Adonis). Distance-based redundancy analysis (db-RDA) and variation partitioning were performed to assess the effect of age and ESBL-status on the broiler's microbial caecal composition using the functions dbrda and varPart from the vegan v.2.5-7 package. dbRDA models were tested for multicollinearity using the variance inflation factors (VIF) with the function vic.cca from the vegan v.2.5-7 package (Oksanen et al., 2020).

\section{ESBL-Ec prevalence}

Prevalences with their associated 95\% confidence intervals based on the 2.5 th and 97.5 th percentiles were estimated for each time point. Confidence intervals were calculated using the Clopper-Pearson binomial method [33] from the prevalence $v$. 0.4.0 package [34]. Prevalence was calculated, and trends in ESBL-Ec prevalence were tested with a chi-squared test for trends in proportions using the function prop.trend.test from the stats v.3.6.2 package.

\section{Declarations}

\section{Ethics approval and consent to participate}


Ethical approval for this study's design was granted through the animal ethical commission at Wageningen University and Research from the Dutch central committee animal experiments in compliance with Dutch legislation, application number AVD4010020171486.

\section{Consent for publication}

Not applicable.

\section{Availability of data and materials}

The datasets generated and/or analysed during the current study are available in the Figshare repository, https://figshare.com/articles/dataset/Data and analyses used in the research paper Succession in the caecal microbiota of developing broilers colonised by Extendedspectrum -lactamase-producing Escherichia coli /16611091

\section{Competing interests}

The authors declare that they have no competing interests.

\section{Funding}

This work was funded by the Dutch Ministry for Agriculture, Nature and Food Quality (grants WOT-01002-003.11 and WOT-01-002-016) and the European Union's Horizon 2020 research and innovation program (grant agreement No 773830). SJ was funded by the German Research Foundation (DFG FZT 118).

\section{Authors' contributions}

JvdG, DC, GB, KV and MB contributed to the design of the work. JvdG, DC, DS and GB performed the sample collection. ICR, TBG, DS and SJ contributed to the data generation and analysis. ICR, TBG, SJ, $\mathrm{KV}, \mathrm{AdV}$ and $\mathrm{MB}$ prepared the manuscript. All authors have revised and approved the submitted version.

\section{Acknowledgements}

The authors thank Arie Kant and Stephanie Vastenhouw for their technical support.

\section{References}

1. Saliu EM, Vahjen W, Zentek J. Types and prevalence of extended-spectrum beta-lactamase producing Enterobacteriaceae in poultry [Internet]. Anim. Heal. Res. Rev. Cambridge University Press; 2017 [cited 2021 Jan 4]. p. 46-57. Available from: https://pubmed.ncbi.nlm.nih.gov/28641596/

2. Van Boeckel TP, Brower C, Gilbert M, Grenfell BT, Levin SA, Robinson TP, et al. Global trends in antimicrobial use in food animals. Proc Natl Acad Sci U S A [Internet]. National Academy of Sciences; 2015 [cited 2019 Feb 20];112:5649-54. Available from: http://www.ncbi.nlm.nih.gov/pubmed/25792457 
3. Liebana E, Carattoli A, Coque TM, Hasman H, Magiorakos A-P, Mevius D, et al. Public Health Risks of Enterobacterial Isolates Producing Extended-Spectrum -Lactamases or AmpC -Lactamases in Food and FoodProducing Animals: An EU Perspective of Epidemiology, Analytical Methods, Risk Factors, and Control Options. Clin Infect Dis [Internet]. Oxford Academic; 2013 [cited 2021 Jan 14];56:1030-7. Available from: https://academic.oup.com/cid/article-lookup/doi/10.1093/cid/cis1043

4. Bradford PA. Extended-spectrum $\beta$-lactamases in the 21st century: Characterization, epidemiology, and detection of this important resistance threat. Clin. Microbiol. Rev. 2001. p. 933-51.

5. Peirano G, Pitout JDD. Extended-Spectrum $\beta$-Lactamase-Producing Enterobacteriaceae: Update on Molecular Epidemiology and Treatment Options [Internet]. Drugs. Springer International Publishing; 2019 [cited 2021 Jun 30]. p. 1529-41. Available from: https://doi.org/10.1007/s40265-019-01180-3

6. Gniadkowski M. Evolution and epidemiology of extended-spectrum $\beta$-lactamases (ESBLs) and ESBLproducing microorganisms [Internet]. Clin. Microbiol. Infect. Blackwell Publishing Ltd.; 2001 [cited 2021 Jan 14]. p. 597-608. Available from: http://www.lahey.org/studies/

7. Carattoli A. Plasmids and the spread of resistance. Int. J. Med. Microbiol. 2013. p. 298-304.

8. Oakley BB, Lillehoj HS, Kogut MH, Kim WK, Maurer JJ, Pedroso A, et al. The chicken gastrointestinal microbiome. FEMS Microbiol Lett. 2014;360:100-12.

9. Sood U, Gupta V, Kumar R, Lal S, Fawcett D, Rattan S, et al. Chicken Gut Microbiome and Human Health: Past Scenarios, Current Perspectives, and Futuristic Applications [Internet]. Indian J. Microbiol. Springer; 2020 [cited 2020 Feb 28]. p. 2-11. Available from: http://www.ncbi.nlm.nih.gov/pubmed/32089569

10. Rychlik I. Composition and function of chicken gut microbiota [Internet]. Animals. MDPI AG; 2020 [cited 2021 Jul 18]. p. 103. Available from: www.mdpi.com/journal/animals

11. Seidlerova Z, Kubasova T, Faldynova M, Crhanova M, Karasova D, Babak V, et al. Environmental impact on differential composition of gut microbiota in indoor chickens in commercial production and outdoor, backyard chickens. Microorganisms. MDPI AG; 2020;8.

12. Tanikawa T, Shoji N, Sonohara N, Saito S, Shimura Y, Fukushima J, et al. Aging transition of the bacterial community structure in the chick ceca. Poult Sci. Elsevier; 2011;90:1004-8.

13. Jurburg SD, Brouwer MSM, Ceccarelli D, van der Goot J, Jansman AJM, Bossers A. Patterns of community assembly in the developing chicken microbiome reveal rapid primary succession. Microbiologyopen [Internet]. John Wiley \& Sons, Ltd; 2019 [cited 2019 May 4];e821. Available from: http://doi.wiley.com/10.1002/mbo3.821 
challenge dose of plasmid-mediated extended-spectrum $\beta$-lactamase and AmpC $\beta$-lactamase producing Escherichia coli on time-until-colonization and level of excretion in young broilers. Vet Microbiol. 2019;

15. Jiménez-Belenguer A, Doménech E, Villagrá A, Fenollar A, Ferrús MA. Antimicrobial resistance of Escherichia coli isolated in newly-hatched chickens and effect of amoxicillin treatment during their growth. Avian Pathol [Internet]. 2016 [cited 2020 Jan 27];45:501-7. Available from: http://www.tandfonline.com/doi/full/10.1080/03079457.2016.1168515

16. Richards P, Fothergill J, Bernardeau M, Wigley P. Development of the Caecal Microbiota in Three Broiler Breeds. Front Vet Sci [Internet]. Frontiers; 2019 [cited 2019 Oct 25];6:201. Available from: https://www.frontiersin.org/article/10.3389/fvets.2019.00201/full 17. Ocejo M, Oporto B, Hurtado A. 16S rRNA amplicon sequencing characterization of caecal microbiome composition of broilers and free-range slow-growing chickens throughout their productive lifespan. Sci Rep [Internet]. Nature Publishing Group; 2019 [cited 2019 Oct 25];9:2506. Available from: http://www.nature.com/articles/s41598-019-39323-x

18. Anjum M, Madsen JS, Nesme J, Jana B, Wiese M, Jasinskyte D, et al. Fate of CMY-2-encoding plasmids introduced into the human fecal microbiota by exogenous escherichia coli. Antimicrob Agents Chemother [Internet]. American Society for Microbiology; 2019 [cited 2020 Nov 24];63. Available from: https://doi.org/10.1128/AAC.02528-18.

19. Wong MHY, Liu L, Yan M, Chan EWC, Chen S. Dissemination of IncI2 plasmids that harbor the blaCTX-M element among clinical Salmonella isolates. Antimicrob Agents Chemother [Internet]. American Society for Microbiology; 2015 [cited 2021 Jul 26];59:5026-8. Available from: /pmc/articles/PMC4505288/

20. Rozwandowicz M, Brouwer MSM, Fischer J, Wagenaar JA, Gonzalez-Zorn B, Guerra B, et al. Plasmids carrying antimicrobial resistance genes in Enterobacteriaceae. J Antimicrob Chemother [Internet]. Oxford University Press; 2018 [cited 2019 Jan 21];73:1121-37. Available from: https://academic.oup.com/jac/article/73/5/1121/4822282

21. Takeshita N, Watanabe T, Ishida-Kuroki K, Sekizaki T. Transition of microbiota in chicken cecal droppings from commercial broiler farms. BMC Vet Res. BioMed Central Ltd; 2021;17.

22. Kers JG, Fischer EAJ, Stegeman JA, Smidt H, Velkers FC. Comparison of different invasive and non-invasive methods to characterize intestinal microbiota throughout a production cycle of broiler chickens. Microorganisms [Internet]. MDPI AG; 2019 [cited 2021 Jul 27];7. Available from: /pmc/articles/PMC6843853/

23. Ducarmon QR, Zwittink RD, Willems RPJ, Verhoeven A, Nooij S, Klis FRM van der, et al. Asymptomatic gut colonization by extended-spectrum beta-lactamase-producing. bioRxiv [Internet]. Cold Spring Harbor Laboratory; 2021 [cited 2021 Jul 21];2021.05.18.444613. Available from: 
24. Dame-Korevaar A, Kers JG, van der Goot J, Velkers FC, Ceccarelli D, Mevius DJ, et al. Competitive Exclusion Prevents Colonization and Compartmentalization Reduces Transmission of ESBL-Producing Escherichia coli in Broilers. Front Microbiol [Internet]. Frontiers Media S.A.; 2020 [cited 2021 Jun 15];11:2853. Available from: https://www.frontiersin.org/articles/10.3389/fmicb.2020.566619/full

25. Dierikx CM, van Duijkeren E, Schoormans AHW, van Essen-Zandbergen A, veldman K, Kant A, et al. Occurrence and characteristics of extended-spectrum- $\beta$-lactamase- and AmpC-producing clinical isolates derived from companion animals and horses. J Antimicrob Chemother [Internet]. J Antimicrob Chemother; 2012 [cited 2021 Jul 26];67:1368-74. Available from: https://pubmed.ncbi.nlm.nih.gov/22382469/

26. Carattoli A, Bertini A, Villa L, Falbo V, Hopkins KL, Threlfall EJ. Identification of plasmids by PCR-based replicon typing. J Microbiol Methods [Internet]. Elsevier; 2005 [cited 2019 Jan 4];63:219-28. Available from: https://www.sciencedirect.com/science/article/pii/S0167701205001132

27. Liakopoulos A, Geurts Y, Dierikx CM, Brouwer MSM, Kant A, Wit B, et al. Extended-spectrum cephalosporinresistant Salmonella enterica serovar heidelberg strains, the Netherlands. Emerg Infect Dis [Internet]. Centers for Disease Control and Prevention (CDC); 2016 [cited 2021 Jun 16];22:1257-61. Available from: http://dx.doi.org/10.3201/eid2207.151377

28. R Core Team. R: A language and environment for statistical computing [Internet]. Vienna, Austria; 2020. Available from: https://www.r-project.org

29. Callahan BJ, McMurdie PJ, Rosen MJ, Han AW, Johnson AJA, Holmes SP. DADA2: High-resolution sample inference from Illumina amplicon data. Nat Methods [Internet]. Nature Publishing Group; 2016 [cited 2019 Nov 22];13:581-3. Available from: http://www.nature.com/articles/nmeth.3869

30. Quast C, Pruesse E, Yilmaz P, Gerken J, Schweer T, Yarza P, et al. The SILVA ribosomal RNA gene database project: improved data processing and web-based tools. Nucleic Acids Res [Internet]. Narnia; 2012 [cited 2019 Dec 16];41:D590-6. Available from: http://academic.oup.com/nar/article/41/D1/D590/1069277/The-SILVAribosomal-RNA-gene-database-project

31. Lin H, Peddada S Das. Analysis of compositions of microbiomes with bias correction. Nat Commun [Internet]. Nature Research; 2020 [cited 2021 Jun 16];11:1-11. Available from: https://doi.org/10.1038/s41467-020-17041-7

32. Oksanen Jari , Blanchet F. Guillaume, Friendly Michael, Kindt Roeland, Legendre Pierre , McGlinn Dan, Minchin Peter R., O'Hara R. B., Simpson Gavin L., Solymos Peter S, Henry SE and WH. vegan: Community Ecology Package [Internet]. 2020. Available from: https://cran.r-project.org/package=vegan 

prevalence assessment studies. [Internet]. 2015. Available from: http://cran.r-project.org/package=prevalence

Figure legends

Figure 1. ESBL-E.coli prevalence and Escherichia/Shigella relative abundance throughout the broiler production round. ESBL-Ec prevalence in the total broiler population (a). Escherichia/Shigella relative abundance over time ESBL-Ec and ESBL-Ec ${ }^{+}$broilers (b). No differential abundance of Escherichia/Shigella between broilers' groups were observed over time (ANCOM-BC, $p>.05)$.

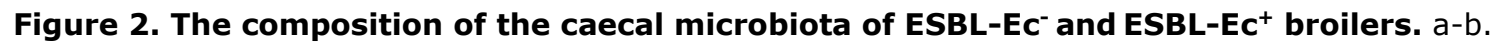
Relative abundance of phyla in ESBL-Ec- and $\mathrm{ESBL}^{-} \mathrm{Ec}^{+}$broilers (a-b). Relative abundance of the top ten genera in ESBL-Ec- and ESBL-Ec $c^{+}$broilers (c-d).

Figure 3. Microbial richness and evenness over time. Observed microbial richness in all broilers over time $(\mathbf{a})$, age significantly predicted microbial richness over time $(p<.001)$. Comparison of microbial richness between $\mathrm{ESBL}-\mathrm{Ec}^{+}$and $\mathrm{ESBL}-\mathrm{Ec}^{-}$broilers (b). The asterisk denotes a $p<.05$ for the Wilcoxon rank-sum test. Comparison of microbial evenness between ESBL-Ec ${ }^{+}$and ESBL-Ec- broilers (c).

Figure 4. Principal coordinate analyses based on Bray Curtis dissimilarity metrics (BC-

PCoA). Changes in the caecal microbial community composition over time (day 0-35). Age explained $14 \%$ of the compositional variation of the caecal microbiome $(B C-d b R D A: F(2,66)=6.47, p=.001)$. ESBL-status explained no variability.

\section{Supplementary files}

Supplementary Figure 1 Relative abundance at the family level. No differential abundance between broiler groups were observed over time (ANCOM-BC, $p>.05)$.

Supplementary Figure 2 Abundance trends of fifteen amplicon sequence variants (ASVs) recovered and associated with Escherichia/Shigella. 
Figures

a

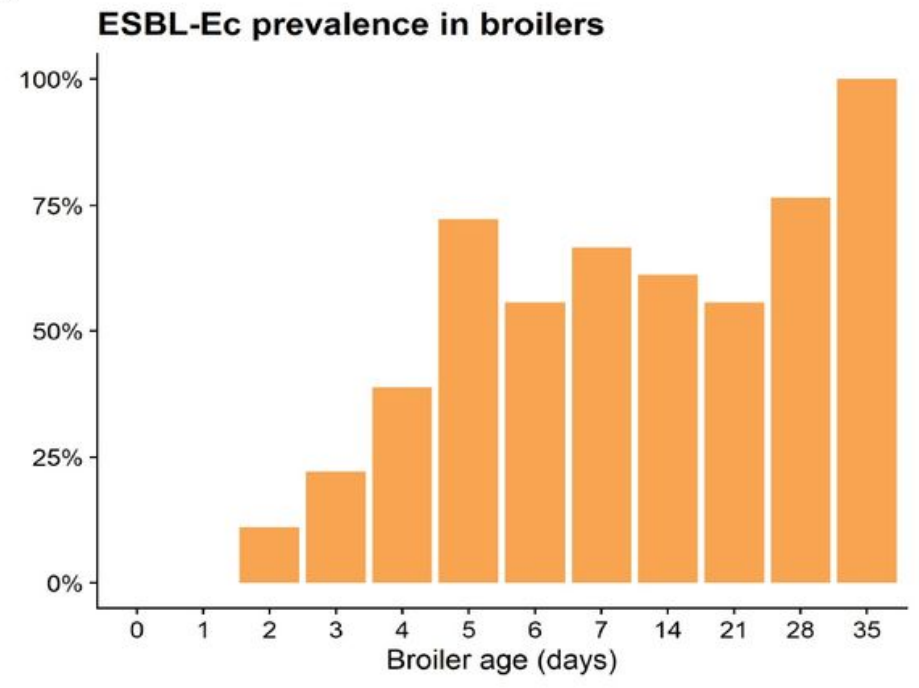

b

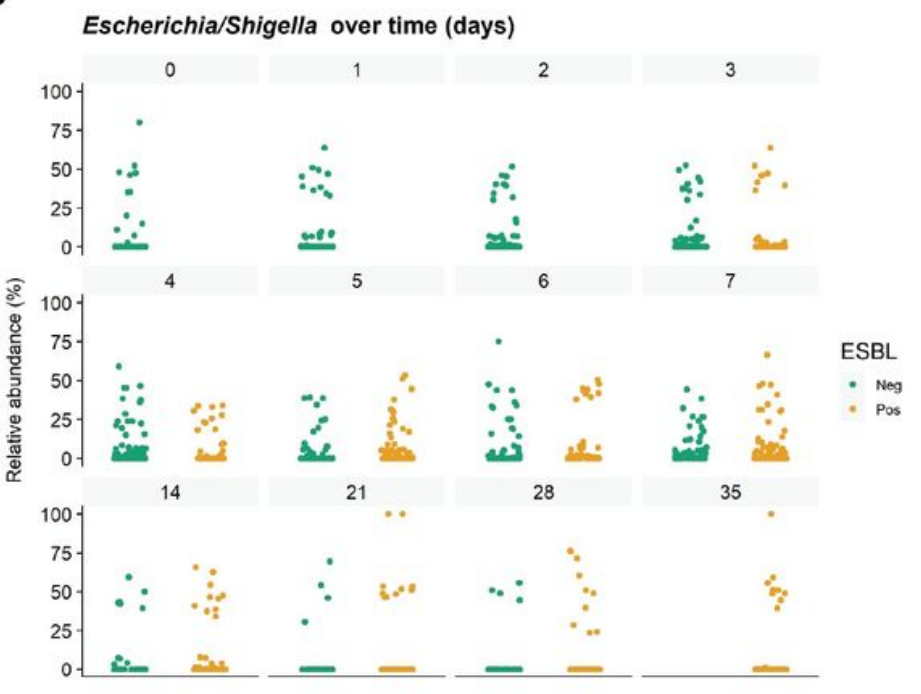

C

Figure 1

ESBL-E.coli prevalence and Escherichia/Shigella relative abundance throughout the broiler production round. ESBL-Ec prevalence in the total broiler population (a). Escherichia/Shigella relative abundance over time ESBL-Ec- and ESBL-Ec+ broilers (b). No differential abundance of Escherichia/Shigella between broilers' groups were observed over time (ANCOM-BC, $p>.05$ ). 
a

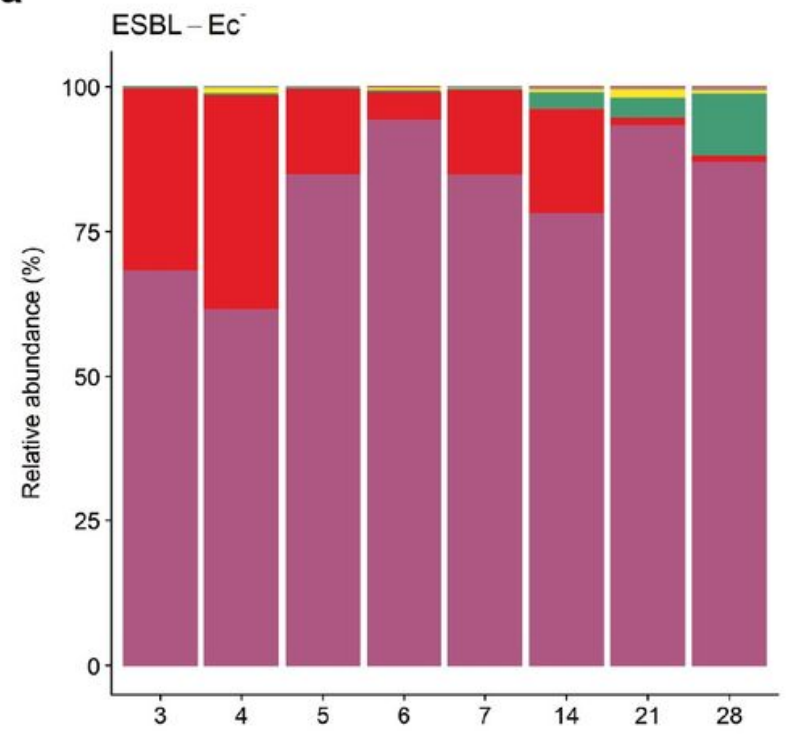

C

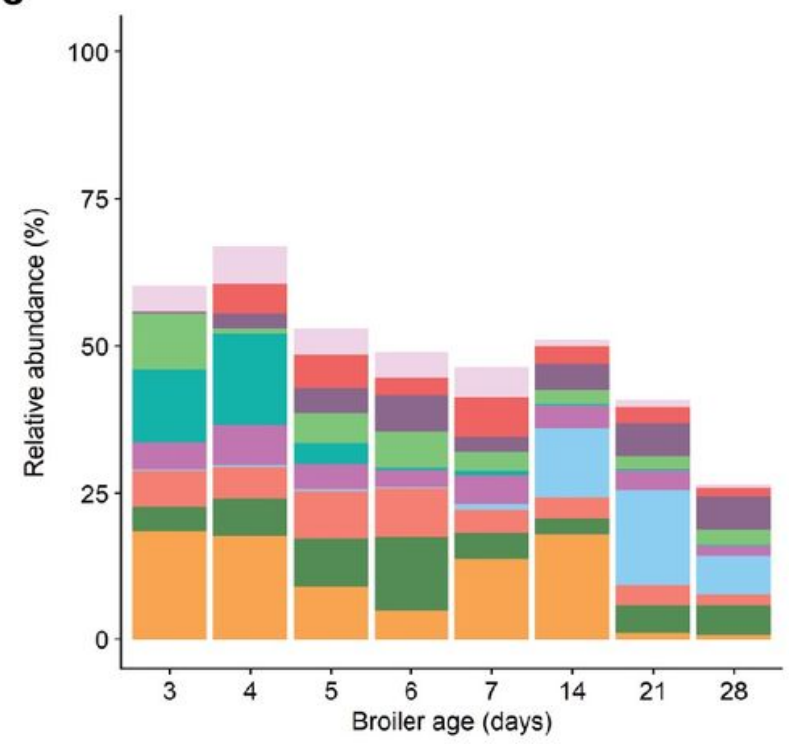

b

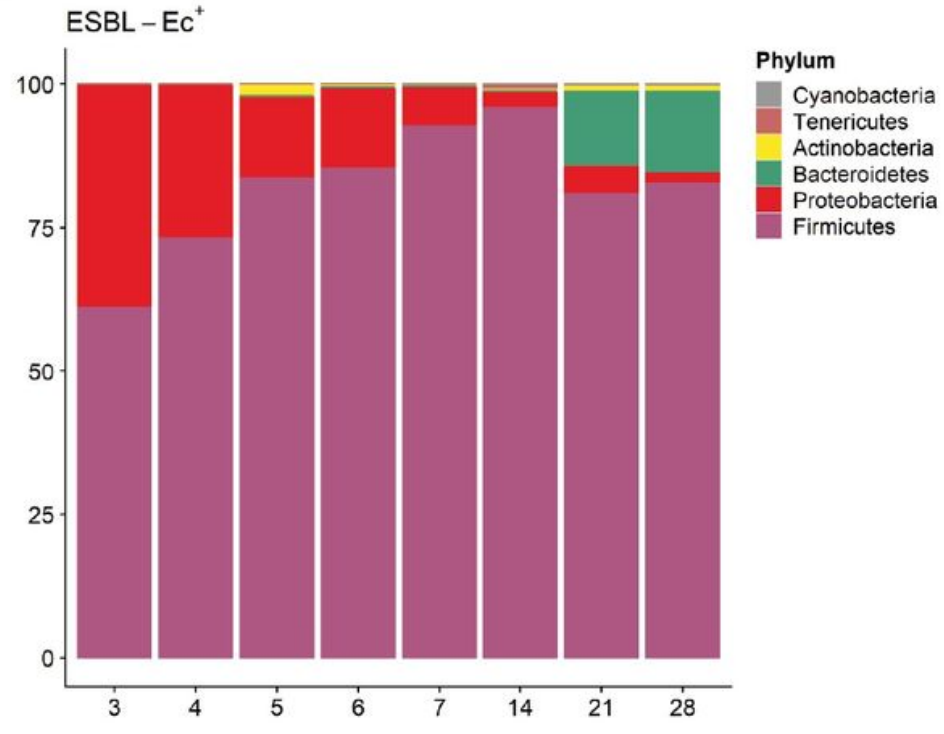

d

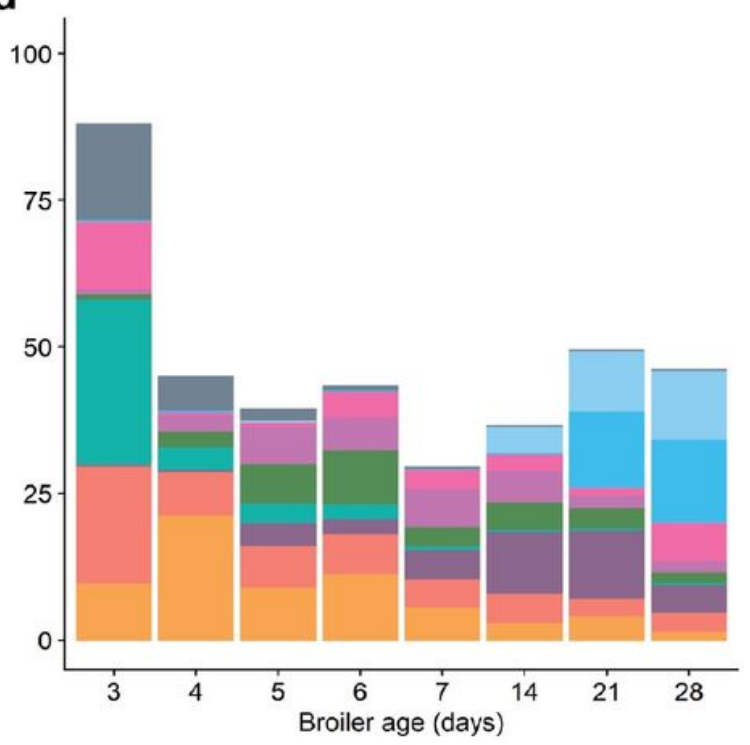

Genus

Enterococcus

Faecalibacterium

Alistipes

Blautia

Butyricicoccus

Flavonifractor

Klebsiella

Subdoligranulum

Lactobacillus

Escherichia/Shigella

\section{Figure 2}

The composition of the caecal microbiota of ESBL-Ec- and ESBL-Ec+ broilers. a-b. Relative abundance of phyla in ESBL-Ec- and ESBL-Ec+ broilers (a-b). Relative abundance of the top ten genera in ESBL-Ec- and ESBL-Ec+ broilers (c-d). 

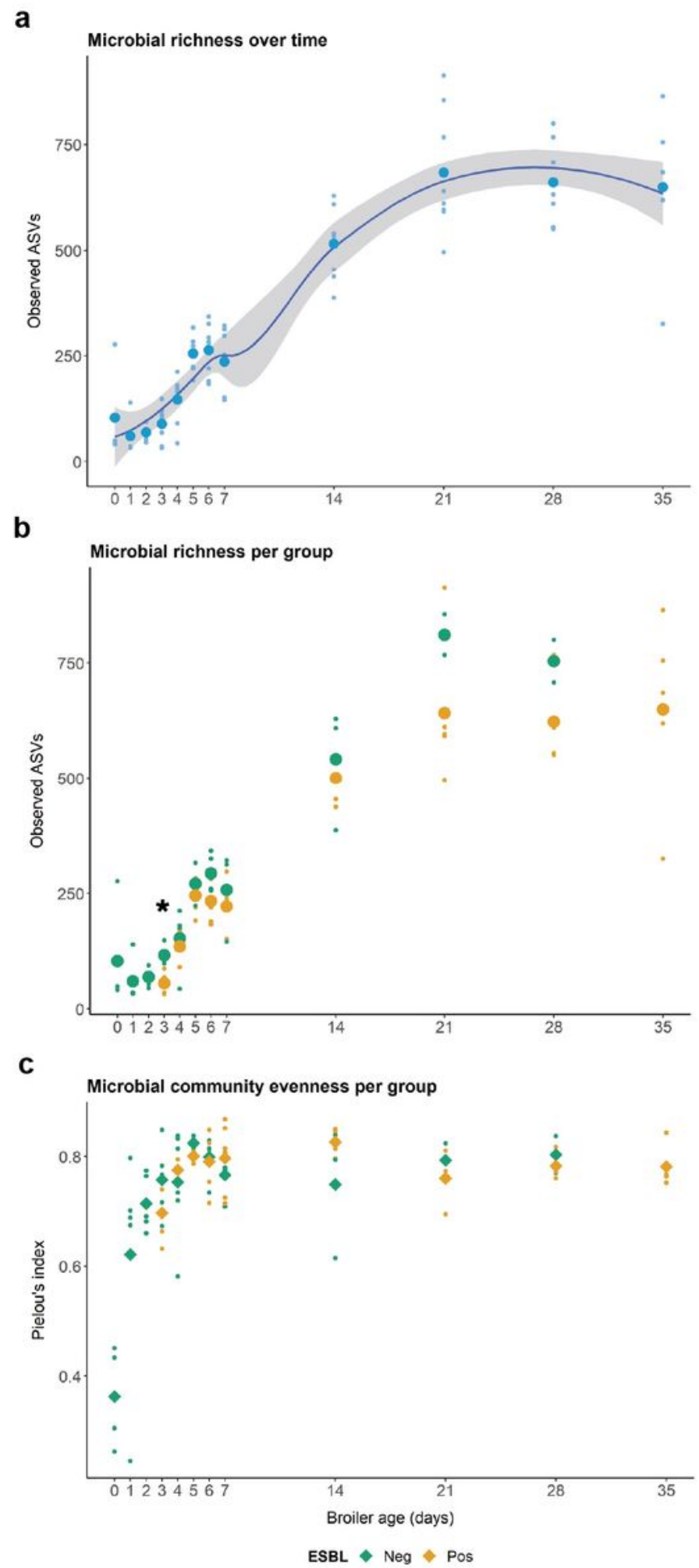

\section{Figure 3}

Microbial richness and evenness over time. Observed microbial richness in all broilers over time (a), age significantly predicted microbial richness over time $(p<.001)$. Comparison of microbial richness between ESBL-Ec+ and ESBL-Ec- broilers (b). The asterisk denotes a $p<.05$ for the Wilcoxon rank-sum test. Comparison of microbial evenness between ESBL-Ec+ and ESBL-Ec- broilers (c). 


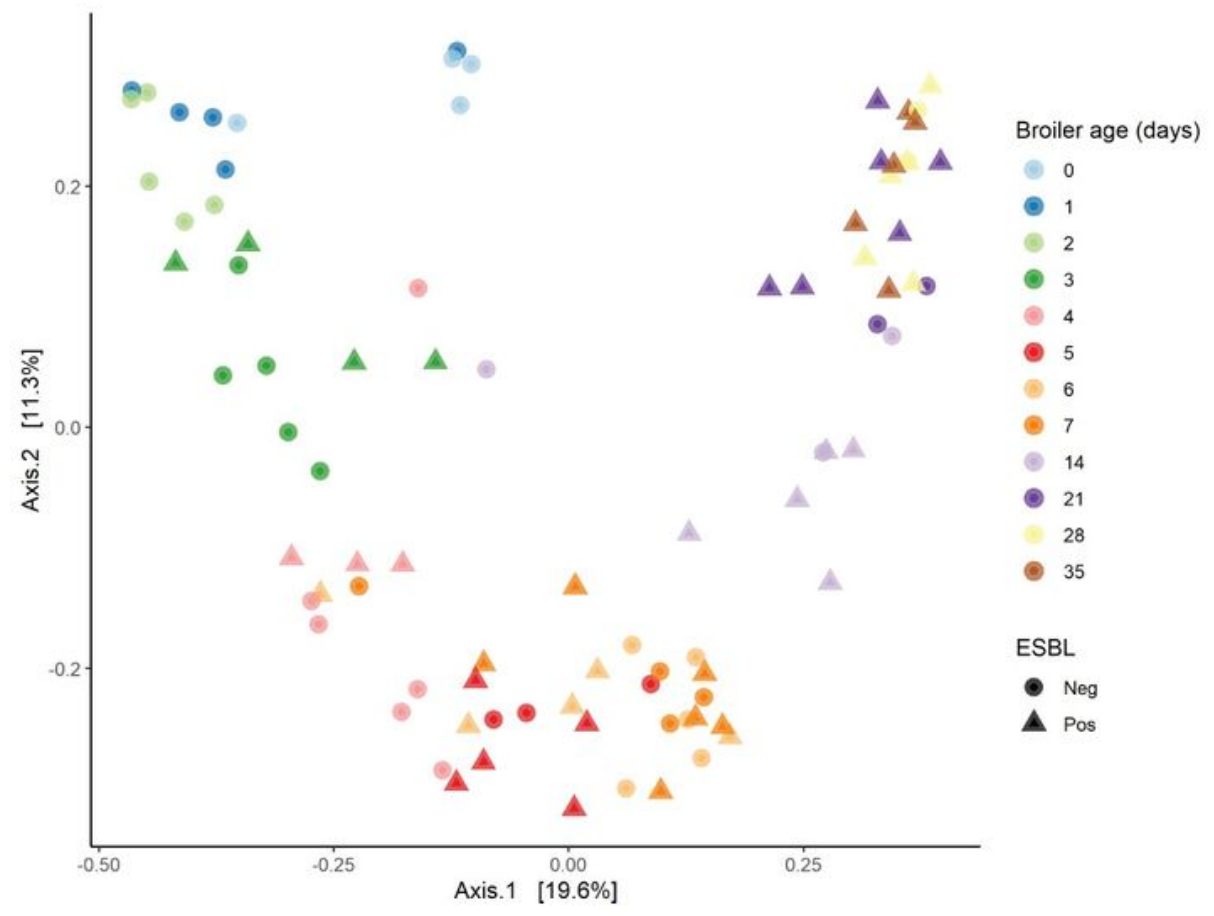

\section{Figure 4}

Principal coordinate analyses based on Bray Curtis dissimilarity metrics (BC- PCoA). Changes in the caecal microbial community composition over time (day 0-35). Age explained $14 \%$ of the compositional variation of the caecal microbiome $(B C-d b R D A: F(2,66)=6.47, p=.001)$. ESBL-status explained no variability. 


\section{Supplementary Files}

This is a list of supplementary files associated with this preprint. Click to download.

- CardenasReyBroilerMicrobiomeSuppltable1.xIsx

- SupplementaryFigure1.png

- SupplementaryFigure2.png 